\title{
Correction to: Development of late-bolting F1 hybrids of Chinese cabbage (Brassica rapa L.) allowing early spring cultivation without heating
}

\author{
Naoko Kitamoto $\cdot$ Kazuhiro Nishikawa Yoshinori Tanimura \\ Shoji Urushibara $\cdot$ Takuya Matsuura $\cdot$ Shuji Yokoi Yoshihito Takahata • \\ Susumu Yui $\mathbb{D}$
}

Published online: 15 January 2018

(C) Springer Science+Business Media B.V., part of Springer Nature 2018

This article was published with an erroneous version of one of the affiliations. Please find the correct affiliations herewith.

S. Yui

Tohoku Agricultural Research Center, National Agriculture and Food Research Organization (NARO),
4 Akahira, Morioka, Iwate 020-0198, Japan (present address) Field Science Center, Faculty of Agriculture, University of Iwate, 1552 Sugo, Takizawa, Iwate, 020-0611, Japan

e-mail: yuis@iwate-u.ac.jp
The original article can be found online at https:// doi.org/10.1007/s10681-017-2079-x.

N. Kitamoto · S. Yokoi · Y. Takahata Laboratory of Plant Breeding, Faculty of Agriculture, University of Iwate, 3-18-8 Ueda, Morioka, Iwate 020-8550, Japan

K. Nishikawa · Y. Tanimura

Sakata Seed Corporation, 358 Uchikoshi, Sodegaura,

Chiba 299-0217, Japan

S. Urushibara · T. Matsuura Iwate Agriculture Research Center, 20-1 Narita, Kitakami, Iwate 024-0003, Japan

\author{
S. Yui $(\bowtie)$ \\ Tohoku Agricultural Research Center, National \\ Agriculture and Food Research Organization (NARO), 4 \\ Akahira, Morioka, Iwate 020-0198, Japan \\ e-mail: yuis@iwate-u.ac.jp \\ Present Address: \\ S. Yui \\ Field Science Center, Faculty of Agriculture, University of \\ Iwate, 1552 Sugo, Takizawa, Iwate 020-0611, Japan
}

